\title{
Cancer of unknown primary ultimately diagnosed as male breast cancer: A rare case report
}

\author{
GEORGIOS RIGAKOS, AMANDA VAKOS, SOTIRIOS PAPADOPOULOS, ANASTASIA VERNADOU, \\ ANTONIOS TSIMPIDAKIS, DIONYSIOS PAPACHRISTOU and EVANGELIA RAZIS
}

Third Department of Medical Oncology, 'Hygeia’ Hospital, 15123 Athens, Greece

Received October 13, 2015; Accepted April 27, 2016

DOI: $10.3892 / \mathrm{mco} .2016 .912$

\begin{abstract}
Cancers of unknown primary (CUP) constitute a significant diagnostic and therapeutic challenge for clinicians and a frequent cause of cancer-related mortality in Western countries. Immunohistochemistry assays are commonly used to identify the primary cancer, but fail in approximately one-third of cases. The identification of the possible origin of CUP is crucial, as it may help select the appropriate treatment options. We herein present the case of a 54-year-old male patient, who presented with lower back pain in June, 2013. Following a thorough investigation, the clinical and pathological findings could not identify the primary cancer, leading towards a misdiagnosis. Ultimately, microRNA testing of the resected spine lesion was able to identify the primary tumor as male breast cancer and allow for optimal treatment of the patient.
\end{abstract}

\section{Introduction}

Cancers of unknown primary origin (CUP) pose a significant challenge for clinicians in terms of identifying the primary cancer and designing an effective treatment strategy for the patients (1). CUPs exhibit an aggressive biological behavior and the prognosis of patients with CUPs is typically significantly poorer compared with that of patients with cancers of known origin, with a median survival of 3-4 months after initial diagnosis (2). While CUPs only comprise $\sim 3-5 \%$ of new cancer cases annually, they constitute the fourth most common cause of cancer-related mortality in Western countries, highlighting the significance of this diagnostic challenge $(3,4)$. Thus, there has been a surge in the development of advanced diagnostic tools for primary tumor identification using molecular testing. Notably, microRNA expression assays have demonstrated a remarkable ability to identify the origin of CUPs (5).

Correspondence to: Dr Sotirios Papadopoulos, Third Department of Medical Oncology, 'Hygeia' Hospital, 4 Erythrou Stavrou Street and Kifissias Avenue, Marousi, 15123 Athens, Greece

E-mail: sotpapado@gmail.com

Key words: cancer of unknown primary, microRNA profile, male breast cancer
MicroRNAs are small, non-coding RNAs that regulate gene expression by RNA degradation or translational inhibition (6). Aberrant microRNAs play a critical role in the development and progression of cancer, making them ideal biomarkers for cancer diagnosis. Multiple studies have reported high sensitivity and accuracy for microRNA assays in diagnosing cases with cancers of known and unknown primary, allowing for their use as a key tool for cancer diagnosis. The case presented herein is a representative example of the possible clinical impact of microRNA assays.

\section{Case report}

A 54 year-old male patient was presented to his orthopedist with low back pain in June, 2013. The patient had no significant past medical history other than mild hypertension and a history of smoking (35 pack-years). An initial magnetic resonance imaging (MRI) examination of the lumbar spine revealed two findings: A mass in the right kidney and a mass in the T9-T10 vertebrae that was impinging on the spinal cord. The patient underwent surgical resection of the vertebral mass at 4 weeks after presentation. Initial pathology identified a metastatic low-grade carcinoma of epithelial origin and the differential diagnosis included renal carcinoma, neuroendocrine carcinoma, melanoma and testicular carcinoma. On immunohistochemistry (IHC), the lesion was strongly positive for cytokeratin AE1/AE3, CAM5.2, epithelial membrane antigen, E-cadherin, melan-A, synaptophysin and placental alkaline phosphatase. Additionally, there was mild and/or focal staining for renal cell carcinoma antigen, CD10, neuron-specific enolase, S100 and human melanoma black 45. The immunostaining for cytokeratin (CK)7, CK20, inhibin, thyroid transcription factor $1, \mathrm{CK} 5 / 6$, gross cystic disease fluid protein 15 , vimentin and CD117, was negative. A second evaluation also raised the possibility of carcinoma of prostatic origin. However, the serum prostate-specific antigen (PSA) levels were within the normal range and IHC for PSA was negative in the tissue sample. Initial evaluation with computed tomography (CT) of the brain, chest, abdomen and pelvis and a bone scan revealed multiple bone lesions, two enlarged mediastinal lymph nodes, a block of enlarged para-aortic lymph nodes and a mass located in the upper pole of the right kidney, sized $11 \times 13.5 \times 12.5 \mathrm{~cm}$. The kidney mass exhibited no significant contrast uptake; following resection, it was found to be a benign cyst. 


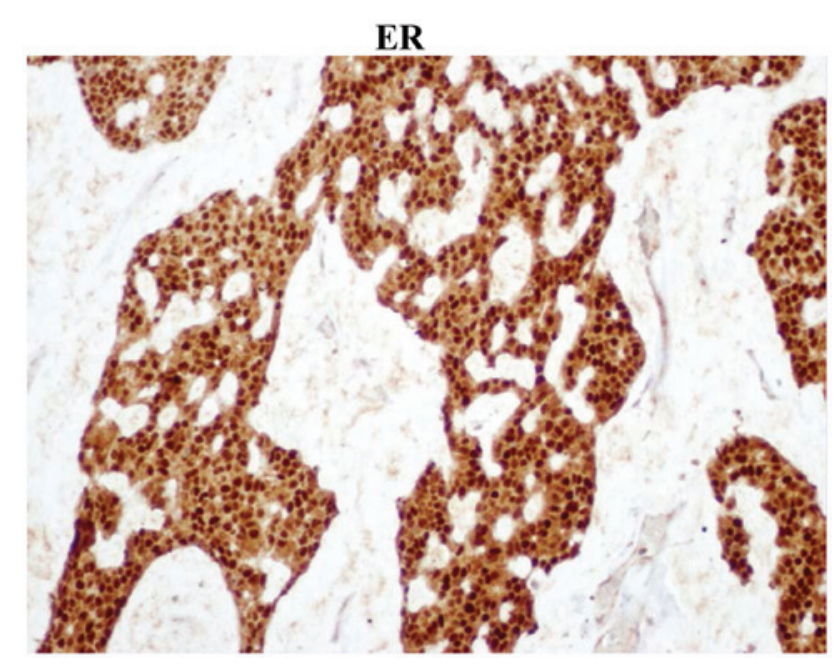

Figure 1. Immunohistochemical staining for estrogen receptor (ER) (magnification, $\mathrm{x} 100)$.

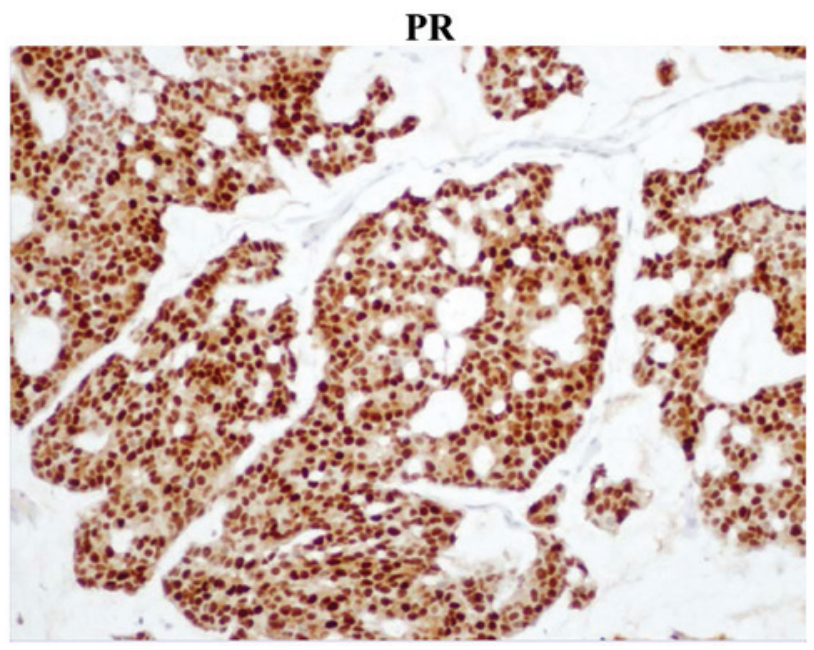

Figure 2. Immunohistochemical staining for progesterone receptor (PR) (magnification, x100)

A positron emission tomography (PET)-CT, ordered in late September, revealed a relatively mild radioactive uptake in the subcutaneous area of the right shoulder girdle [standardized uptake value $(\mathrm{SUV})=3.1$, and a significantly higher uptake in several bones (SUVmax $=9.3$ ). Physical examination of the patient revealed a soft, hairy, pigmented skin lesion on the right anterior axillary line. An MRI scan of the right shoulder was ordered on the 18th of October, and the mass appeared as a subcutaneous lesion with homogeneous contrast uptake with an associated group of lymph nodes. As the primary cancer remained unknown, the microRNA Rosetta Cancer Origin test (Rosetta Genomics Ltd., Philadelphia, PA, USA) was ordered 16 weeks after presentation. The test results were received 18 weeks after presentation and identified the tissue as breast cancer, with a sensitivity of $90 \%$. The axillary mass was then resected (20 weeks after presentation) and pathology identified that mass as a malignant neoplasm similar to the mass removed from the lumbar area. IHC was $100 \%$ positive for estrogen receptor (ER) and progesterone receptor (PR) and positive for human epidermal growth factor receptor 2 (HER2) (Figs. 1-3).

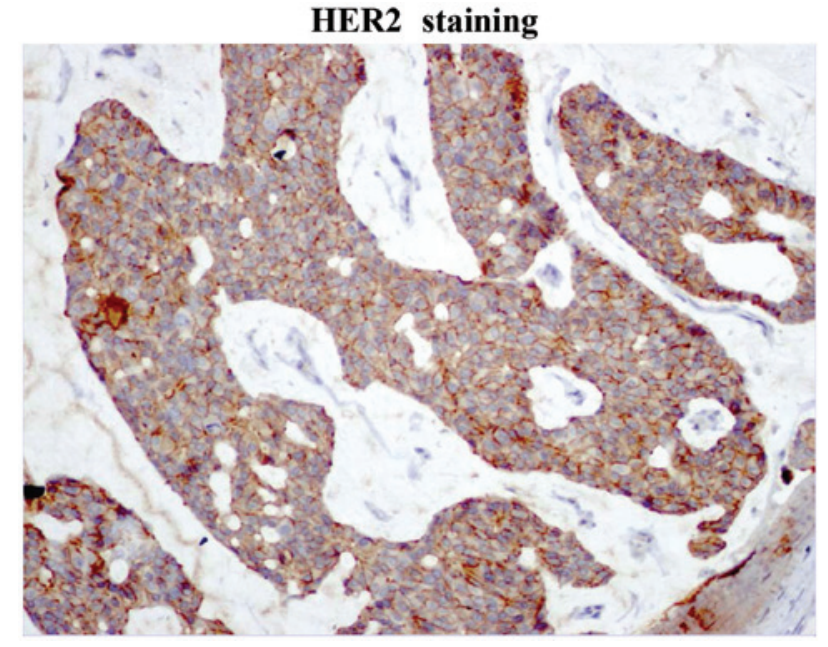

Figure 3. Immunohistochemical staining for human epidermal growth factor receptor 2 (HER2) (magnification, x100).

The patient was treated for breast cancer with capecitabine and trastuzumab as the first-line regimen; following relapse on left iliac and right pubic bone, the regimen was changed to vinorelbine and trastuzumab. The patient responded well and proceeded to maintenance trastuzumab and tamoxifen. On December 30, 2014, treatment was switched to letrozole and trastuzumab, due to an increase in serum marker levels (carcinoembryonic antigen and carbohydrate antigen 15-3) that was followed by the appearance of a skin nodule right by the scar of the axillary mass resection. The nodule was subsequently removed and histology showed local relapse of the known neoplasm. The patient is completely asymptomatic and continues on letrozole and trastuzumab with stable bone disease ever since, while the relevant tumor markers are within normal limits. The patient currently remains on maintenance letrozole and trastuzumab, has stable disease (bone lesions alone) and a good overall clinical status. Last follow-up was on March 16, 2016.

Patient informed consent was obtained for the publication of the case details.

\section{Discussion}

Identifying the primary cancer is crucial in selecting the optimal therapeutic strategy for the patients. When clinical and pathological methods fail to identify the primary tumor, treatment is often delayed and inaccurately targeted, contributing to the generally very poor prognosis of patients with CUPs. IHC is the most widely used tool for the diagnosis of several types of cancer, but fails to identify the tissue of origin in $>30 \%$ of the cases (7). Furthermore, IHC may be biased based on the patient's presentation and past medical history and the interpretation of the results may be subjective, further signifying the need for alternative cancer diagnostic tests. A number of studies have demonstrated that microRNA profiling may be useful for solving the diagnostic problem posed by CUPs, with agreement to final diagnosis for microRNA testing ranging from $84 \%$ to as high as $92 \%$, depending on the study (8-10). MicroRNAs are small, non-coding RNAs of 17-25 nucleotides in length that play an essential regulatory role in protein translation and 

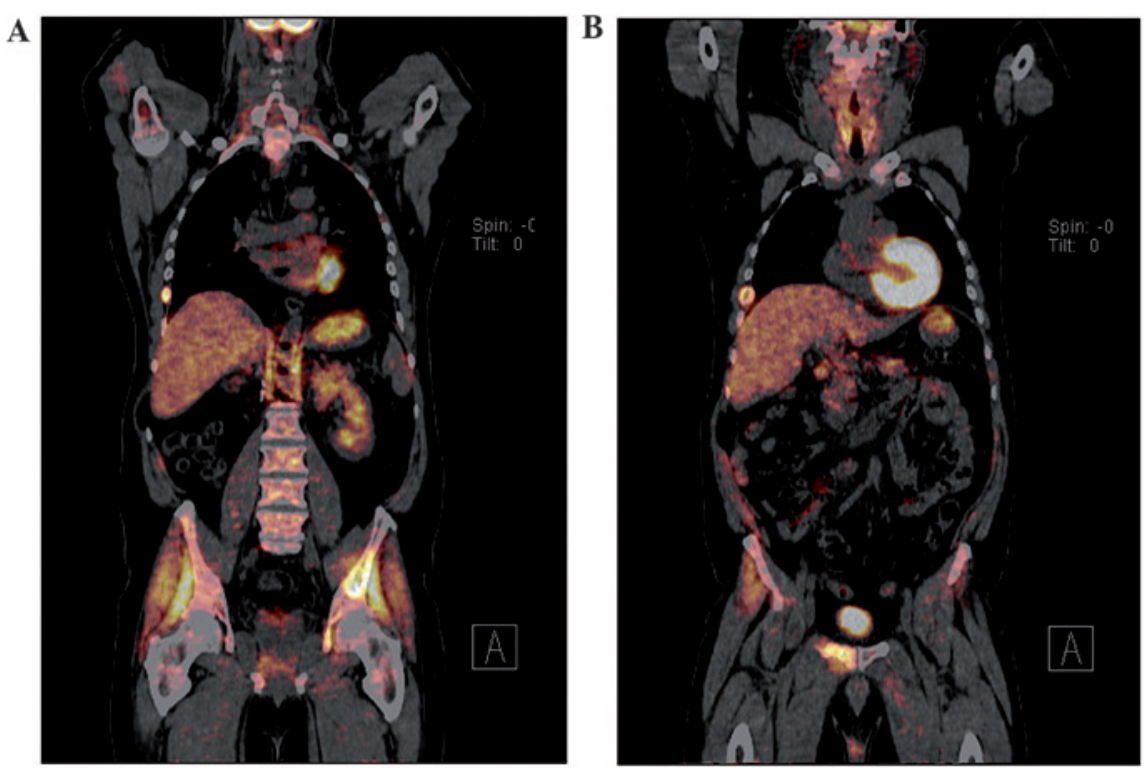

Figure 4. Macroscopic appearance of the skin lesion in (A) righ pubic and (B) left iliac lesion.

expression (6). Since their discovery in 1993, microRNAs have been implicated in a number of major cell processes and have been mapped to areas of the genome prone to deletions, mutations and amplifications (6). Their ability to act as oncogenes or tumor suppressors, combined with their unique tissue pattern expression in malignancies, makes microRNAs ideal cancer biomarkers and provides the basis for their use as a diagnostic tool (11).

In the present case, microRNA testing was crucial, as the clinical and pathological findings did not lead to a diagnosis of breast cancer for a number of reasons. First, the pathological examination of the vertebral mass did not raise the suspicion of breast cancer, so this was not included in the differential diagnosis. Second, while the MRI and PET-CT imaging found the axillary mass to be suspicious, clinical evidence regarding the origin of the mass pointed towards the skin rather than the breast. The clinical evaluation of the skin lesion was also more consistent with skin cancer, taking into consideration the appearance and coloration of the mass (Fig. 4). Finally, the patient was male, with no family history of breast cancer. This is significant, as male breast cancer (MBC) is a rare entity, constituting $<1 \%$ of all breast cancers and $<1 \%$ of cancers in men (12). MBC is also notably more difficult to diagnose compared with female breast cancer. Male patients tend to be older than female patients at diagnosis, and have higher-stage disease, with more extensive lymph node involvement, contributing to the high mortality rate of $\operatorname{MBC}(13,14)$. MicroRNA testing proved to be key in identifying the origin of the primary cancer, when the clinicopathological picture was leading towards a misdiagnosis. Further examination revealed that the tumor was $\mathrm{ER}+, \mathrm{PR}+$ and $\mathrm{HER} 2+$, indicating that the patient had a particularly good prognosis and could be treated with trastuzumab, as well as several specific targeted therapies, which would not have been applied had it not been for the microRNA test.

MicroRNA profiling as a diagnostic test offers several advantages, the most important being that the assay is objective and unbiased. Samples may be profiled using quantitative polymerase chain reaction technology or, more recently, a custom microRNA array, and classification of tumor types relies on specific bioinformatics algorithms. The microarray is both powerful and flexible, it is able to profile thousands of transcripts and newly identified microRNAs may easily be incorporated into an updated test design. Furthermore, in terms of the feasibility of testing, samples of microRNAs may be safely stored for significant periods of time in formalin-fixed paraffin-embedded tissues and, when extracted, still exhibit similar profiles to microRNA samples from fresh tissue (15). Multiple studies have repeatedly demonstrated high test sensitivity, specificity and reproducibility, and the turnaround time for profiles is between 7 and 10 days, rendering this a reliable and practical diagnostic test. Furthermore, microRNA screening for early cancer diagnosis shows promising potential with changes in microRNA profiles implicated in early or pre-tumor development (6). However, there are challenges to microRNA testing regarding CUPs. First, the accuracy of testing cannot be directly validated, as there is no primary cancer identified to be used as reference; thus, cancers with similar profiles may be easily confused as being of the same origin $(5,8)$. In addition, microRNA assessment may not always agree with the clinical and pathological diagnosis, with a previous study reporting a disagreement rate of $16 \%$ (8). Furthermore, the assay itself may produce two different diagnoses, which poses a potential problem for clinicians if the diagnoses differ regarding the optimal course of treatment (8). On the other hand, the test may not be able to predict a result at all if the expression patterns do not closely correspond to those included in the panel. It is of great importance to determine the potential benefit that patients may gain from assigning CUP to a primary tissue of origin. We would expect that assigning a CUP to a primary would be of great benefit for such patients; however, evidence thus far does not strongly support this hypothesis. A recent prospective study by Hainsworth et al (16), in which molecular techniques were used to identify 
the primary, demonstrated a median survival of 12.5 months for the group of patients in whom the CUP was assigned a primary tissue of origin, in contrast to the group of patients who were treated as CUP, in whom the median survival was 10.8 months. Under the light of the currently available data, the European Society for Medical Oncology and National Comprehensive Cancer Network guidelines are quite conservative regarding the use of molecular tools in the differential diagnosis of CUP, and they advise careful selection of the population considered as candidate for such diagnostic tools (level of evidence, 2B). Finally, while most recent tests may screen for 42 different primaries, they do not screen for all cancer origins (8).

MicroRNA profiling may be quite costly, although the cost-benefit ratio may ultimately be favorable, as several unnecessary tests may be avoided. For all the abovementioned reasons, clinicians must consider all data relative to each individual case, in order to determine the optimal course of treatment for each patient.

The case presented herein highlights the efficacy of using microRNA analysis of tumor tissue samples to diagnose CUPs. As a diagnostic tool, microRNA profiles may be very useful in identifying the primary tissue of origin and, thus, improving treatment and outcome for CUP patients.

\section{References}

1. Varadhachary GR and Raber MN: Cancer of unknown primary site. N Engl J Med 371: 757-765, 2014

2. Riihimäki M, Thomsen H, Hemminki K, Sundquist K and Hemminki K: Comparison of survival of patients with metastases from known versus unknown primaries: Survival in metastatic cancer. BMC Cancer 13: 36, 2013.

3. Pavlidis N and Fizazi K: Cancer of unknown primary (CUP). Crit Rev Oncol Hematol 54: 243-250, 2005.

4. Pavlidis N, Briasoulis E, Hainsworth J and Greco FA: Diagnostic and therapeutic management of cancer of an unknown primary. Eur J Cancer 39: 1990-2005, 2003.

5. Ferracin M, Pedriali M, Veronese A, Zagatti B, Gafà R, Magri E, Lunardi M, Munerato G, Querzoli G, Maestri I, et al: MicroRNA profiling for the identification of cancers with unknown primary tissue-of-origin. J Pathol 225: 43-53, 2011.

6. Iorio MV and Croce CM: MicroRNA dysregulation in cancer: Diagnostics, monitoring and therapeutics. A comprehensive review. EMBO Mol Med 4: 143-159, 2012.

7. Anderson GG and Weiss LM: Determining tissue of origin for metastatic cancers: Meta-analysis and literature review of immunohistochemistry performance. Appl Immunohistochem Mol Morphol 18: 3-8, 2010.
8. Varadhachary GR, Spector Y, Abruzzese JL, Rosenwald S, Wang H, Aharonov R, Carlson HR, Cohen D, Karanth S, Macinskas $\mathrm{J}$, et al: Prospective gene signature study using microRNA to identify the tissue of origin in patients with carcinoma of unknown primary. Clin Cancer Res 17: 4063-4070, 2011.

9. Pentheroudakis G, Pavlidis N, Fountzilas G, Krikelis D, Goussia A, Stoyianni A, Sanden M, St Cyr B, Yerushalmi N, Benjamin H, et al: Novel microRNA-based assay demonstrates 92\% agreement with diagnosis based on clinicopathologic and management data in a cohort of patients with carcinoma of unknown primary. Mol Cancer 12: 57, 2013.

10. Rosenwald S, Gilad S, Benjamin S, Lebanony D, Dromi N, Faerman A, Benjamin H, Tamir R, Ezagouri M, Goren E, et al: Validation of a microRNA-based qRT-PCR test for accurate identification of tumor tissue origin. Mod Pathol 23: 814-823, 2010.

11. Li M, Li J, Ding J, He M and Cheng SY: MicroRNA and cancer. AAPS J 12: 309-317, 2010.

12. Rizzolo P, Silvestri V, Tommasi S, Pinto R, Danza K, Falchetti M, Gulino M, Frati P and Ottini L: Male breast cancer: Genetics, epigenetics, and ethical aspects. Ann Oncol 24 (Suppl 8): viii75-viii82, 2013.

13. Schneider S and Sariego J: Male breast cancer presenting as an axillary mass: A case report and literature review. South Med J 102: 736-737, 2009.

14. Korde LA, Zujewski JA, Kamin L, Giordano S, Domchek S, Anderson WF, Bartlett JM, Gelmon K, Nahleh Z, Bergh J, et al: Multidisciplinary meeting on male breast cancer: Summary and research recommendations. J Clin Oncol 28: 2114-2122, 2010.

15. Rosenfeld N, Aharonov R, Meiri E, Rosenwald S, Spector Y, Zepeniuk M, Benjamin H, Shabes N, Tabak S, Levy A, et al: MicroRNAs accurately identify cancer tissue origin. Nat Biotechnol 26: 462-469, 2008.

16. Hainsworth JD, Rubin MS, Spigel DR, Boccia RV, Raby S, Quinn R and Greco FA: Molecular gene expression profiling to predict the tissue of origin and direct site-specific therapy in patients with carcinoma of unknown primary site: A prospective trial of the Sarah Cannon research institute. J Clin Oncol 31: 217-223, 2013. 